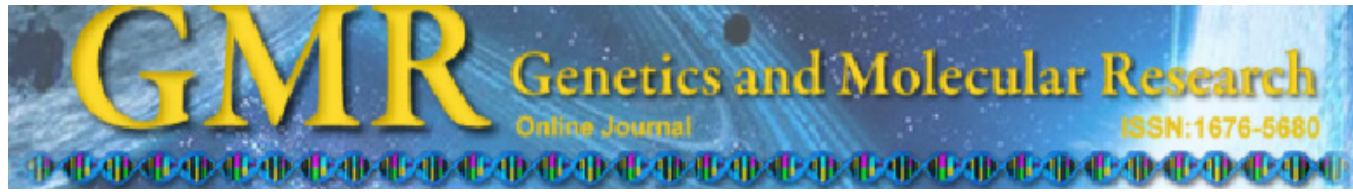

\title{
Molecular characterization and structure analysis of RPL10/QM-like protein from the red drum Sciaenops ocellatus (Sciaenidae)
}

\author{
X. Chen ${ }^{1,3}$, C.-W. Wu ${ }^{2}$, S.-P. Zhong ${ }^{1}$, F.-R. Zeng ${ }^{1}$, J.-S. Zhang ${ }^{2}$, J. Wang ${ }^{1}$ \\ and S.-F. Niu ${ }^{1}$ \\ ${ }^{1}$ State Key Laboratory of Marine Environmental Science, Xiamen University, \\ Xiamen, China \\ ${ }^{2}$ Marine Sciences College, Zhejiang Ocean University, Zhoushan, China \\ ${ }^{3}$ Key Laboratory for Tropical Biological Resources, Hainan University, \\ Haikou, China
}

Corresponding author: J. Wang

E-mail: junw@xmu.edu.cn

Genet. Mol. Res. 10 (2): 576-587 (2011)

Received November 3, 2010

Accepted December 20, 2010

Published April 5, 2011

DOI 10.4238/vol10-2gmr1134

\begin{abstract}
The QM-like gene encodes a ribosomal protein L10. Besides housekeeping roles in protein synthesis, QM-like proteins have multiple extraribosomal functions during cell growth, cell differentiation and apoptosis. We obtained the full-length cDNA of QM-like protein (designated as SoQM) from the salt water game fish Sciaenops ocellatus, using RACE-PCR. The sequence consists of $740 \mathrm{bp}$, encoding 215-amino acid residues with $24.60 \mathrm{kDa}$. The AA sequence of the SoQM protein contains a series of functional motifs that belong to the QM family signature, which is conserved among different species. The SoQM gene contains five introns and six exons. The expression pattern of SoQM as determined by RT-PCR indicated that SoQM mRNA was expressed in all tissues tested, including brain, gill, head-kidney, intestine, stomach, heart, spleen, blood, muscle, and gonads. The phylogenetic tree constructed with MEGA 4.0 showed that SoQM clusters together with that of other fish. It was found that the sequences of the SoQM gene are highly conserved, suggesting the fundamental and
\end{abstract}


critical functions of SoQM in S. ocellatus. The three-dimensional structure of the SoQM protein core domain (4 169) was predicted by the Swiss-Model program. Compared with QM proteins in other species, the main structure of SoQM protein was conserved, while the C-terminal domain was different from other QM-like proteins. Prediction of the three-dimensional structure of SoQM would provide valuable insight into the molecular basis of protein function, allowing an effective design of experiments, such as site-directed mutagenesis, studies of disease-related mutations or structure-based design of specific inhibitors.

Key words: SoQM; Molecular cloning; Structure analysis; Red drum

\section{INTRODUCTION}

The QM gene was originally isolated as a putative Wilms' tumor suppressor (Dowdy et al., 1991). The higher expression level of the QM gene in nontumorigenic Wilms' microcell hybrid cells than in the tumorigenic parental cell line suggested that the QM gene product was a putative tumor suppressor. Increasing evidence suggested that besides housekeeping roles in protein synthesis, QM-like proteins have multiple extraribosomal functions during cell growth, cell differentiation and apoptosis (Marty et al., 1993; Green et al., 2000; Lillico et al., 2002). It was found that the sequences of QM genes were highly conserved, suggesting the fundamental and critical functions of QM in eukaryotic organisms. The product of the QM-like gene is ribosomal protein L10 (RPL10) with a molecular mass of 24 to $26 \mathrm{kDa}$, and the protein has two basic domains possibly to bind to DNA and an acidic domain involved in the activation of transcription (Farmer et al., 1994). To date, more and more studies have focused on the structure of the QM-like protein to try to elucidate the molecular mechanism of the protein function (Dresios et al., 2006; Korostelev et al., 2006; Nishimura et al., 2008). The mutation of GRC5/QSR1 resulted in a defect in protein synthesis, growth and cell division arrest, and abnormalities of the actin cytoskeleton and mitochondrial respiration in yeast (Tron et al., 1995; Koller et al., 1996). QM has been determined, corresponding to residues 34182 of the full-length 214 amino acids (Nishimura et al., 2007, 2008). Deletion of C-terminal residues of Escherichia coli ribosomal protein L10 caused the loss of binding of one L7/L12 dimer: ribosomes with one L7/L12 dimer were active (Griaznova and Traut, 2000). Moreover, relationships of L10 to other proteins have also been reported (Monteclaro and Vogt, 1993; Imafuku et al., 1999; Park and Jeong, 2006; Chen et al., 2006), although their significance is still less obvious.

To our knowledge, QM-like gene has not been well documented in marine fish. In this paper, we reported the identification of a cDNA encoding a QM-like protein from Sciaenops ocellatus (designated as SoQM), a marine fish of high economic impact in China. Furthermore, we analyzed the sequence of the gene and its expression pattern in different tissues of $S$. ocellatus by reverse transcription-polymerase chain reaction (RT-PCR). Finally, we predicted the three-dimensional structures of this molecule to provide valuable insight into the molecular basis of protein function, allowing an effective design of experiments, such as site-directed mutagenesis, studies of disease-related mutations or the structure-based design of specific inhibitors. 


\section{MATERIAL AND METHODS}

\section{Isolation of total RNA and synthesis of cDNA}

S. ocellatus (average length $300 \mathrm{~mm}$ and weight $650 \mathrm{~g}$ ) were purchased from the market of Daxue Road, Xiamen, China. Total RNA was extracted from $70 \mathrm{mg}$ gill tissue of $S$. ocellatus with Trizol (Invitrogen, USA) according to the manufacturer protocol. Total RNA, 500 $\mathrm{ng}$, was used as the template and the primer was Oligo $\mathrm{dT}_{(18)}$ to synthesize the first cDNA using the RevertAid ${ }^{\mathrm{TM}} \mathrm{H}$ Minus First Strand cDNA Synthesis kit (Fermentas, USA). Two primers (QMP_forward and QMP_reverse, Table 1), according to highly conserved regions of known QM-like genes, were used in RT-PCR. Amplification was performed in a 50- $\mu \mathrm{L}$ mixture containing $5 \mu \mathrm{L}$ Taq buffer (10X), $4 \mu \mathrm{L}$ dNTP mix (2.5 mM each), $4 \mu \mathrm{L} \mathrm{MgCl}_{2}(25 \mathrm{mM}), 0.25 \mu \mathrm{L}$ Taq polymerase $(5 \mathrm{U} / \mu \mathrm{L})$ (TaKaRa, Japan), $2 \mu \mathrm{L}$ forward and reverse primers (each $10 \mu \mathrm{M}$ ), $1.5 \mu \mathrm{L}$ cDNA template and $33.25 \mu \mathrm{L}$ sterile double-distilled $\mathrm{H}_{2} \mathrm{O}$. Finally, the PCR product was electrophoresed on a $1.2 \%$ agarose gel, stained with ethidium bromide and photographed with an ImageQuant Scanner (GE Healthcare, USA) using DL2000 as size marker. Plasmid DNA from at least six independent clones was sequenced using an ABI 3730 Sequencer (Applied Biosystems, USA).

Table 1. The sequences and functions of primers used in this study.

\begin{tabular}{lll}
\hline Name & Sequence $\left(5^{\prime} \rightarrow 3^{\prime}\right)$ & Function \\
\hline QMP_forward & TTCMAGKTYCCTGGACRCCA & Amplification of the cDNA fragment \\
QMP_reverse & CTGSCGNCCWGMGAACTTG & \\
Tdaptor3' & GACTCGAGTCGACATAGA(T) $)_{17}$ & 3' RACE-PCR \\
Adaptor3' & GACTCGAGTCGACATAG & \\
QM3'_1 & TGCGGTAAGGATGGTTT & \\
QM3'_2 & AGACTGGAATGCGTGGTGCT & 5' RACE-PCR \\
QM5'_T & CCAGTGAGCAGAGTGACGAGGACTCGAGCTCAAGC(T) ${ }_{17}$ & \\
QM5'_O & CCAGTGAGCAGAGTGACG & \\
QM5'_I & GAGGACTCGAGCTCAAGC & \\
QM5'_1 & CCAGTCTGGAGCCTATCAGCT & Real-time PCR, RT-PCR \\
QM5'_2 & GCTCTGGAGGCTGCCCGTAT & \\
qRT_forward & CCTGCGGTAAGGACGGTTC & \\
qRT_reverse & AAAGCACCACGCATTCCAGT & \\
Actin_forward & GCCCAGAGCAAGAGGGGTA & Amplification of the genomic sequence \\
Actin_reverse & ATCACCGGAGTCCATGACA & \\
GP_forward1 & CCTCCATTAGTTCGCAACCATG & \\
GP_reverse1 & GTACTTGTTAGCACAGATACGG & \\
GP_forward2 & GGGCAGGAAGAAGGCCAAGGT & \\
GP_reverse2 & CTGCAGCAGCTCTAGACGGCATG & \\
\hline
\end{tabular}

RACE $=$ rapid amplification of cDNA ends; $\mathrm{PCR}=$ polymerase chain reaction.

\section{Rapid amplification of cDNA ends (RACE)}

The primers were designed based on the initial SoQM gene sequence for 5' and 3' RACE-PCR (QM5'_1, QM5'_2, QM3'_1, and QM3'_2) (Table 1). cDNA was synthesized from total RNA extracted as described above with a First Strand cDNA Synthesis kit (GE Healthcare, USA), following manufacturer instructions. For 5' RACE-PCR, the primers containing adaptor, including QM5'_T, QM5'_O and QM5'_I (Table 1), were designed according to Sambrook and Russell (2001) and Scotto-Lavino et al. (2006). 


\section{Isolation of DNA and cloning of genomic gene}

Genomic DNA was isolated from muscle of S. ocellatus (average weight $150 \mathrm{~g}$ ) with the Wizard Genomic DNA purification kit (Promega, USA) as described by Buonocore et al. (2003). Genomic DNA was resuspended in 30 100 $\mu \mathrm{L}$ sterilized water. The quality of the DNA was checked by running $1 \mu \mathrm{L}$ DNA on an ethidium bromide-stained agarose gel $(0.5 \%, \mathrm{w} / \mathrm{v})$. Based on the $S$. ocellatus cDNA sequence, several primers (Table 1$)$ were constructed to obtain the entire SoQM gene. The cycling protocol was 1 cycle of $94^{\circ} \mathrm{C}$ for 5 min and 35 cycles of $94^{\circ} \mathrm{C}$ for $45 \mathrm{~s}, 50^{\circ} \mathrm{C}$ for $45 \mathrm{~s}$ and $72^{\circ} \mathrm{C}$ for $45 \mathrm{~s}$, followed by 1 cycle of $72^{\circ} \mathrm{C}$ for $10 \mathrm{~min}$, using the Taq PCR Master Mix kit (Qiagen, German). Genomic DNA, $10 \mathrm{ng}$, was used for each PCR. PCR products were visualized, sequenced and analyzed as described above.

\section{Expression analysis of SoQM in vitro}

To investigate tissue expression of SoQM, RT-PCR was carried out using total RNA from brain, gill, head-kidney, intestine, stomach, heart, spleen, blood, muscle, and gonad of $S$. ocellatus. Using the Trizol reagent, the first cDNA was synthesized as described above. Primers (Table 1) were designed based on the SoQM cDNA sequences presented in this study. $\beta$-actin mRNA, representing a housekeeping gene, was amplified to provide an internal control for the QM-like gene expression analysis. The PCR amplification conditions were 1 cycle of $94^{\circ} \mathrm{C}$ for $5 \mathrm{~min}$ and 35 cycles of $94^{\circ} \mathrm{C}$ for $30 \mathrm{~s}, 60^{\circ} \mathrm{C}$ for $40 \mathrm{~s}$ and $72^{\circ} \mathrm{C}$ for $30 \mathrm{~s}$, followed by 1 cycle of $72^{\circ} \mathrm{C}$ for $5 \mathrm{~min}$. Amplified products were analyzed on a $2.0 \%$ agarose gel stained with ethidium bromide, and the amount of each product was quantified visually.

\section{Analysis of sequence alignment and construction of phylogenetic tree}

Based on the amino acid sequences of QM proteins, a phylogenetic tree was constructed using the MEGA 4.0 program with the neighbor-joining method (NJ) (Tamura et al., 2007). Phylogenetic tree calculation was done using Clustal X1.83 based on a sequence distance method and utilizing the NJ algorithm of Saitou and Nei (1987). The nucleotide sequences used in this paper were available from GenBank: Canis lupus familiaris, Ctenopharyngodon idella, Danio rerio, Entamoeba histolytica, Gallus gallus, Homo sapiens, Ictalurus punctatus, Ornithorhynchus anatinus, Pinctada fucata, Pseudosciaena crocea, Rattus norvegicus, Saccharomyces cerevisiae, Solea senegalensis, and Xenopus tropicalis.

\section{Prediction of three-dimensional structure}

The predicted three-dimensional structure of SoQM was created using the template model of 2zkrh from the C. familiaris Swiss-Model procedure (http://swissmodel. expasy.org//SWISS-MODEL.html) (Arnold 2006). The quality of the resulting model was assessed using the atomic non-local environment assessment (ANOLEA) mean force potential (Melo and Feytmans, 1998; Kiefer et al., 2009). 


\section{RESULTS AND DISCUSSION}

\section{Analysis of SoQM cDNA sequence}

The full-length cDNA from S. ocellatus (GenBank accession No. FJ455762) designated as SoQM is 740-bp, including a 19-bp 5' terminal untranslated region (UTR), 648-bp coding region and 73-bp 3' UTR. The 648-bp coding region was found to encode a protein with 215- amino acid residues (Figure 1) analyzed by ORF Finder. The molecular mass of the protein was $24.60 \mathrm{kDa}$ and the isoelectric point was 10.138. The SoQM protein consisted of 45 strongly basic $(+)$ amino acids (K,R), 18 strongly acidic(-) amino acids (D,E), 65 hydrophobic amino acids (A,I,L,F,W,V), and 41 polar amino acids (N,C,Q,S,T,Y), analyzed by DNAstar. There were 2 basic domains (1 33, 150 175), possibly to bind to DNA, and 1 acidic domain, involved in activation of transcription (55 76) as in other species (Farmer et al., 1994). Analyzed by the PredictProtein program, the SoQM protein contained 2 acylamidation sites (1 4 MGRR, 36 39 LGRK), 2 protein kinase C (PKC) phosphorylation sites (SVR, 137 139; SKK, 168 170), 1 N-myristoylation site (GMRGAF, 114 119), 2 amidation sites (MGRR, 1 4; LGRK, 36 39), and 1 ribosomal protein L10 signature (RGAFGKPQGTVARV, 116 129) (Figure 1). We compared the sequence with that of Trypanosoma brucei, which indicated that QM-like protein from T. brucei has only one putative PKC phosphorylation site (137 139) (Lillico, 2002), by which PKC may introduce phosphate into QM and therefore regulate its binding to c-Jun (Inada et al., 1997). The average hydrophobicity of the polypeptide analyzed by (http://www.expasy.org/cgi-bin/protscale.pl) was -0.517 , which suggested that SoQM was probably an insoluble protein. Analyzed by (http://www.expasy.org/tools/protparam.html), the instability index was 46.05, indicating the protein to be unstable. The aliphatic index was 67.16. No N-terminal signal sequence was found using the SignalP program, indicating that the SoQM was not a secretory protein.

\section{Analysis of SoQM gene structure}

PCR amplification of genomic DNA, using the primers deduced from the SoQM cDNA sequence, gave overlapping products revealing the complete gene nucleotide sequence. PCR performed with GP_forward1 and GP_reverse 1 (Table 1) yielded a 1948-bp product. PCR performed with GP_forward2 and GP_reverse2 (Table 1) yielded a 1453-bp product. The whole contiguous nucleotide sequence comprises 2913 bp (GenBank accession No. GQ398776) with 6 exons consisting of $42,59,108,139,163$, and $212 \mathrm{bp}$, respectively, and 5 introns consisting of $944,345,416,118$, and 367 bp, respectively (Figure 2). All introns started with the typical "GT" nucleotide sequence and ended with the usual "AG" sequence (Figure 1). With regard to the genomic sequence of SoQM, the sizes of the introns were quite variable between different species, and the exon sizes were much more conserved than the intron ones.

\section{Alignment and phylogenetic tree constructed}

To determine the relatedness of the putative SoQM with known QM-like sequences, a multiple alignment of the deduced amino acid sequence of SoQM with other QM family members was performed (Figure 3). Compared to the $\mathrm{N}$-terminus and the middle sequence of 


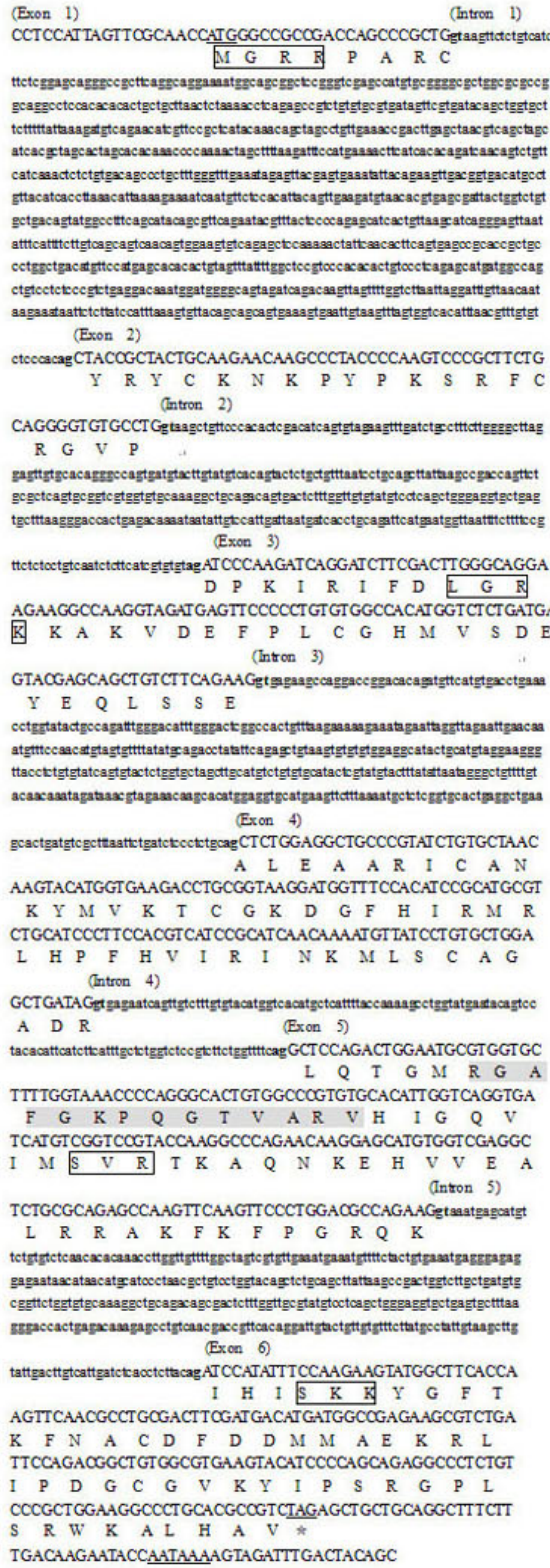

Figure 1. The full-length nucleotide sequence of SoQM with deduced amino acid sequence below. *Indicates the stop codon, and the polyadenylation signal (AATAAA) is underlined. A series of motifs in the putative GcQM polypeptide are boxed, including two acylamidation sites MGRR (1 4) and LGRK (36 39), two protein kinase C phosphorylation sites SVR (137 139) and SKK (168 170), and N-myristoylation site GMRGAF (114 119). In addition, a ribosomal protein $\mathrm{L} 10$ (116 129) signature is shaded. 


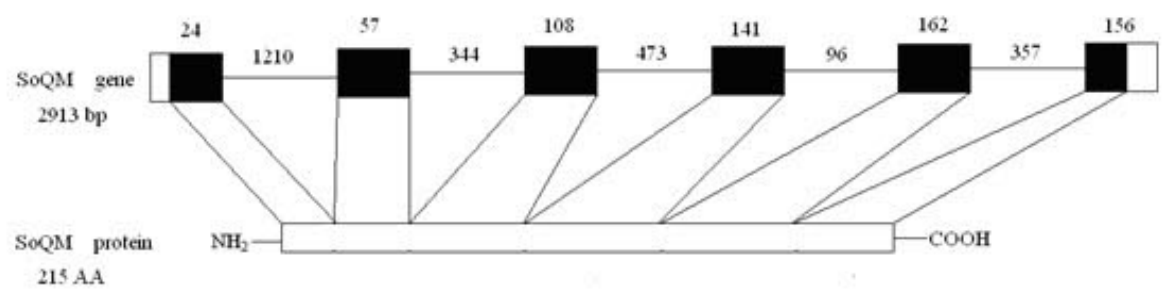

Figure 2. Genomic organization of the SoQM gene. Numbers above the bold and thin lines represent exon and intron length (bp), respectively. The blank boxes are the coding sequences, where the open boxes represent the untranslated region of the mRNA.

the QM-like proteins, the C-terminus sequences were much less conserved. We inferred that difference in the $\mathrm{C}$-terminus sequence between these species could lead to different spatial structures of the C-terminus and play different functions in different organisms. To study the phylogenetic relationship of SoQM gene and that of the other species, a phylogenetic tree was constructed using the MEGA 4.0 program of the NJ method, and the matrix of sequence identities was shown by the DNAStar program. As shown in Figure 4, the tree was composed of three main groups: 1 ) fish (S. ocellatus, $S$. senegalensis, $C$. idella, D. rerio, $P$. crocea, and I. punctatus); 2) mammals (H. sapiens, $R$. norvegicus, C. lupus familiaris, and $O$. anatinus) together with X. tropicalis and G. gallus, and 3) others: molluscs (P. fucata), protozoa (E. histolytica) and ascomycota (S. cerevisiae). Compared with other QM-like protein AA sequences, SoQM protein displayed high homology to other fish-related sequences.

Figure 5 shows that SoQM protein shared high degree of similarity with $P$. crocea (99.1\%), S. senegalensis (98.1\%), C. idella (96.7\%), D. rerio (96.3\%), I. punctatus $(95.3 \%)$, C. lupus familiaris $(91.2 \%)$, H. sapiens $(93.0 \%)$, R. norvegicus $(92.5 \%)$, X. tropicalis $(92.1 \%)$, G. gallus (91.9\%), and O. anatinus (91.6\%), and had a lower homology with P. fucata (76.3\%), S. cerevisiae $(64.2 \%)$ and E. histolytica $(55.7 \%)$.

\section{Analysis of SoQM expression in vitro}

To find clues to the function of SoQM in S. ocellatus in vivo, the tissue-specific expression of SoQM in different tissues, including brain, gill, head-kidney, intestine, stomach, heart, spleen, blood, muscle, and gonads, was examined by RT-PCR. The results showed that SoQM was expressed in all tissues examined. RT-PCR of $\beta$-actin, a housekeeping gene, was also performed to serve as an expression control among different tissues (Figure 6). GcQM from grass carp was expressed in spleen, heart and brain in healthy fish, respectively, while no expression was detectable in head-kidney, liver, intestine, and muscle (Wen et al., 2005). This is probably because of their different habitats.

\section{The prediction of three-dimensional structure of SoQM protein}

The three-dimensional structure of the SoQM protein core domain (residues 4 169) was predicted by the Swiss-Model procedure (http://swissmodel.expasy.org// SWISS-MODEL.html) (Figure 7). The template was 2zkrh from C. familiaris. The sec- 


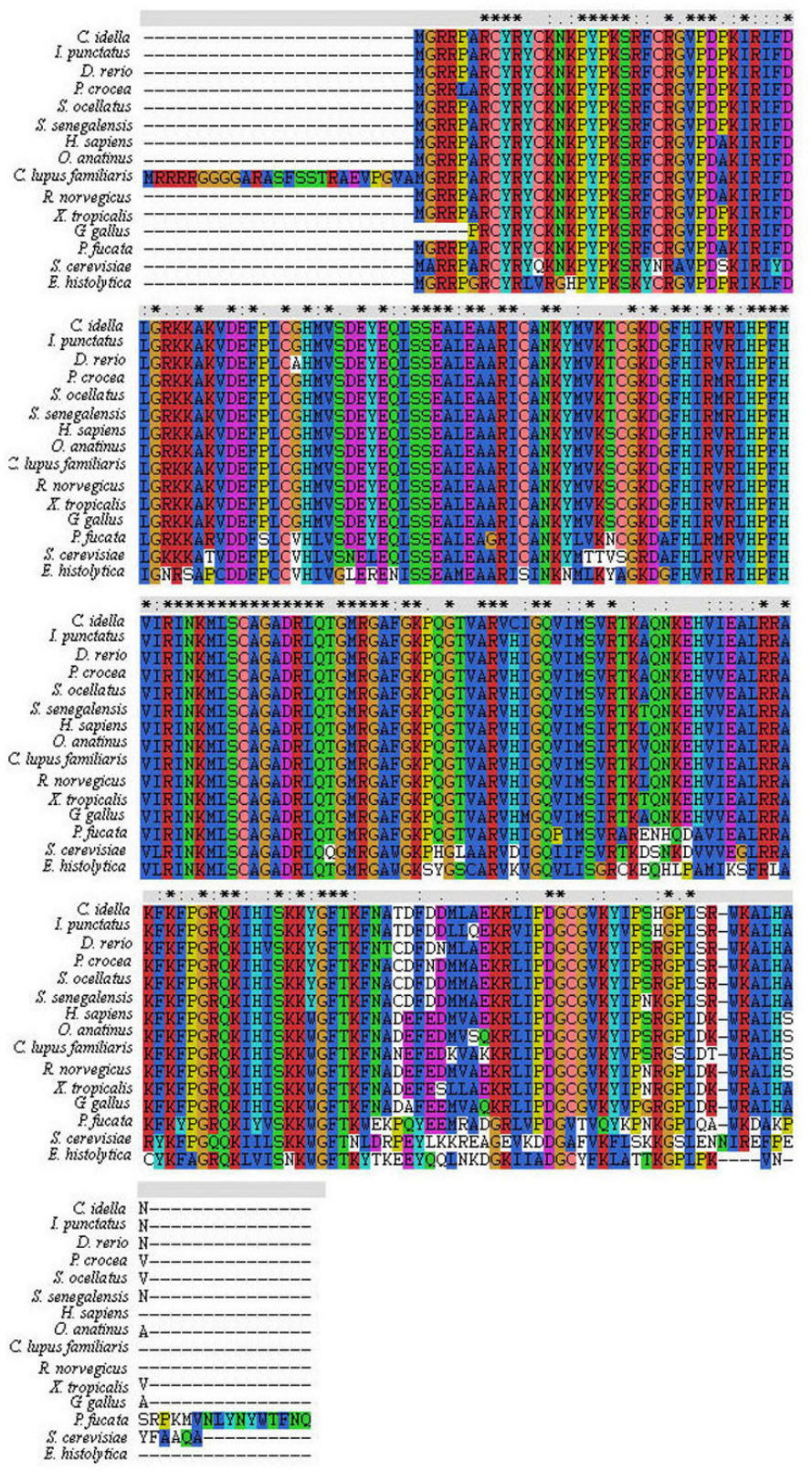

Figure 3. Alignment of the predicted amino acid sequence of the SoQM gene with those of corresponding proteins in other organisms. (*) Indicates identical amino acids; (:) indicates conserved residues. GenBank accession Nos. for these amino acid sequences are as follows: C. idella AAV31599, C. lupus familiaris XP 547794, D. rerio NP 956321, E. histolytica AAL68397, G. gallus, H. sapiens NP_006004, I. punctatus AAK95135, O. anatinus XP_001508298, P. fucata AAQ09228, P. crocea ACU87547, R. norvegicus NP_112362, S. cerevisiae CAA55485, $\bar{S}$. ocellatus this study, S. senegalensis BAF98656, X. tropicalis NP 001004965. 


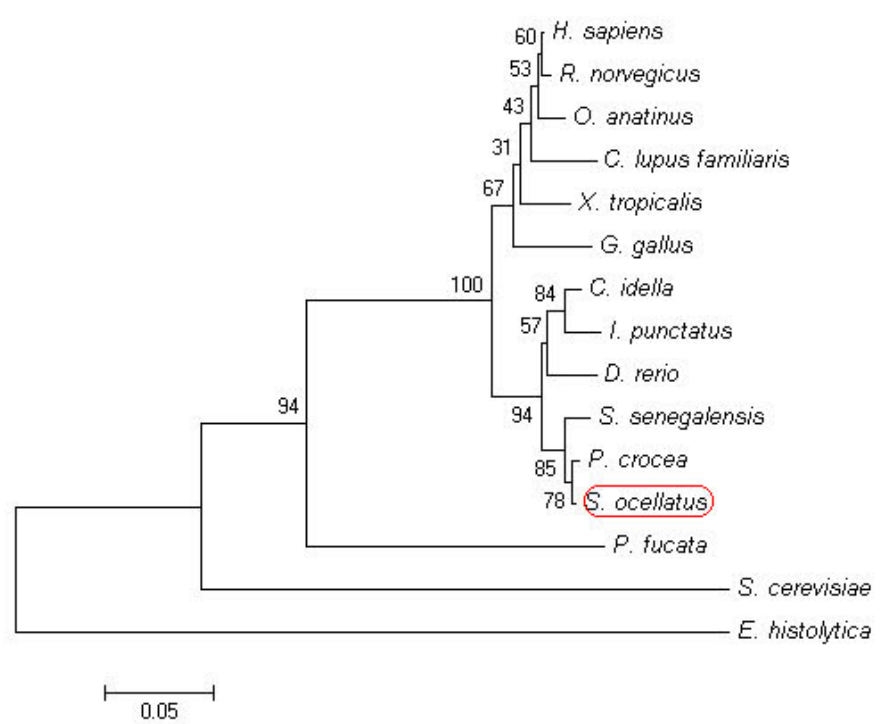

Figure 4. Phylogenetic tree showing the evolutionary relationship between SoQM and other known QM-like proteins. The rooted tree was constructed by the "neighbor-joining" method and was bootstrapped 10,000 times. 0.05 indicates the genetic distance.

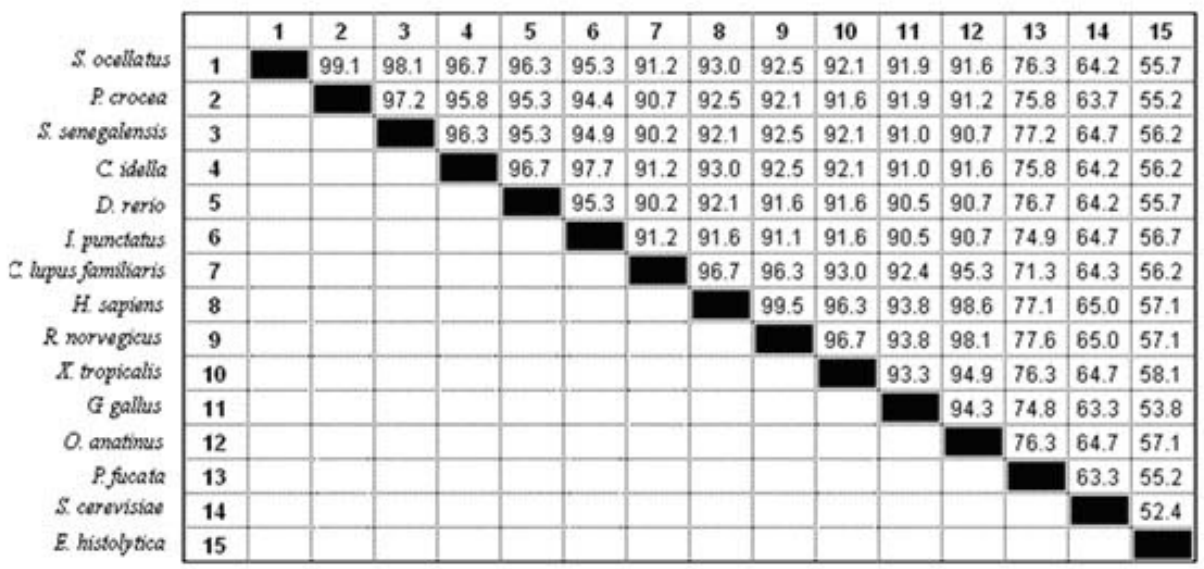

Figure 5. Matrix indicating the percentage identities of the aligned QM-like proteins.

ondary structure element was $\beta 1-\beta 2-\alpha 1-\beta 3-\beta 4-\beta 5-\alpha 2-\beta 6$, identical with that of QM from $H$. sapiens (Nishimura et al., 2008). The SoQM protein shared $95.78 \%$ identity with the template with an E value of $2.24388 \mathrm{e}-87$. There were a few residues different between them. Ala 28, Ser 78, Val 88, Lys 127, Ile 137, Leu 141, and Ile 148 in 2zkrh were replaced by Pro 29, Thr 79, Met 89, Arg 128, Val 138, Ala 142, and Val 149 in the SoQM protein (Figure 7). The results indicated that the SoQM protein core domain contained $2 \alpha$-helices and $6 \beta$-sheets. ANOLEA was used in the Swiss-Model procedure to assess the quality of the model (SoQM 
protein). The ANOLEA value of the residues of the SoQM protein was $<0$, expected for residues 10 23, 48 50, 103 112, 107, 121 123, 146 (Figure 8). According to the protocol, the lower the ANOLEA value, the more accurate the predicted structure of model will be (Kopp and Schwede, 2004, 2006); the predicted structure of the SoQM protein was reasonable. These bioinformatics analyses provided important information for further investigation of SoQM. The prediction of three-dimensional structure of SoQM provides valuable insight into the molecular basis of protein function, allowing an effective design of experiments, such as sitedirected mutagenesis, studies of disease-related mutations or the structure-based design of specific inhibitors (Kopp and Schwede, 2004).

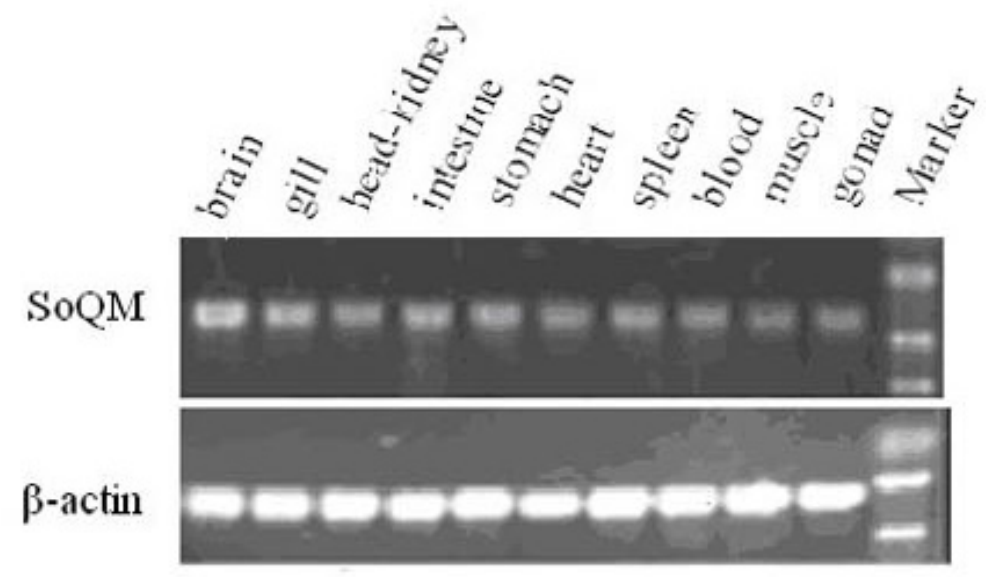

Figure 6. In vitro SoQM expression analysis was performed using cDNAs collected from different tissues as designated. The $\beta$-actin serves as an internal control for the homogeneity of cDNA samples. The SoQM transcripts are expressed in all tissues examined.

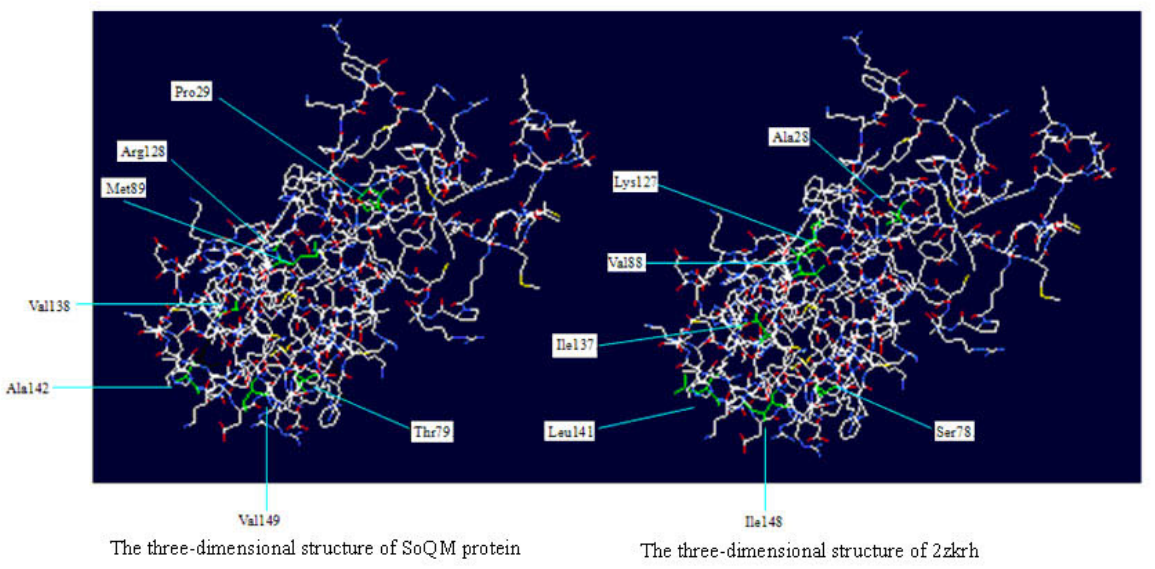

Figure 7. Three-dimensional alignment of the tertiary structures of SoQM and 2zkrh predicted by the Swiss-Model procedure. The different amino acids between SoQM and 2zkrh are noted. 


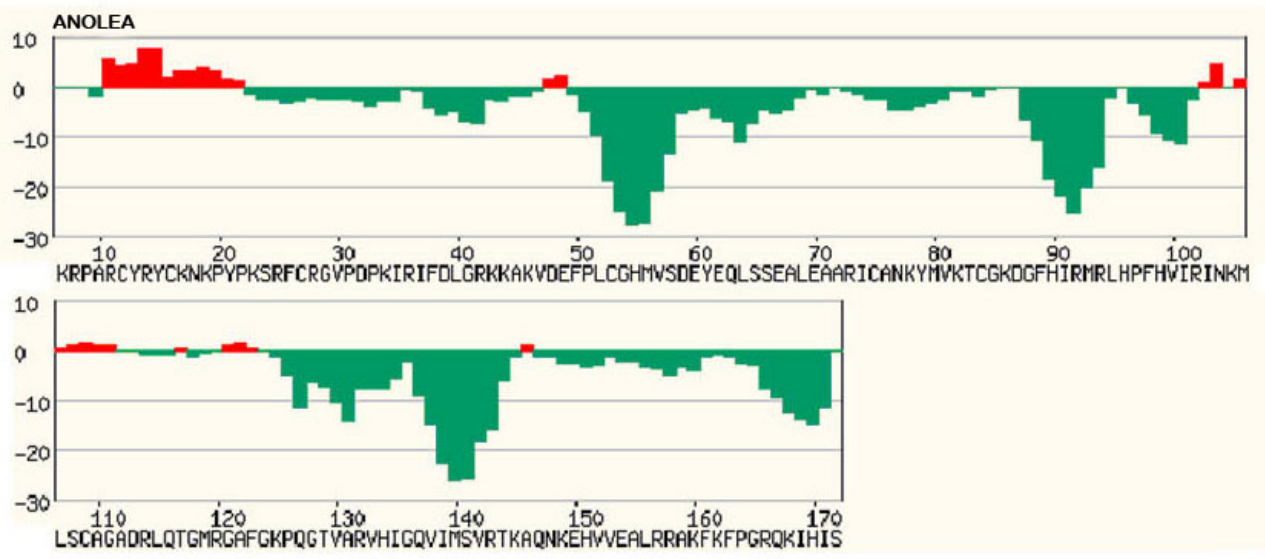

Figure 8. The quality assessment of the tertiary structure of SoQM protein. ANOLEA = atomic non-local environment assessment.

\section{ACKNOWLEDGMENTS}

Research supported by the National High Technology Research and Development Program of China (863 Program) under contract \#2006AA10A405.

\section{REFERENCES}

Arnold K, Bordoli L, Kopp J and Schwede T (2006). The SWISS-MODEL workspace: a web-based environment for protein structure homology modelling. Bioinformatics 22: 195-201.

Buonocore F, Prugnoli D, Falasca C, Secombes CJ, et al. (2003). Peculiar gene organisation and incomplete splicing of sea bass (Dicentrarchus labrax L.) interleukin-1beta. Cytokine 21: 257-264.

Chen C, Wanduragala S, Becker DF and Dickman MB (2006). Tomato QM-like protein protects Saccharomyces cerevisiae cells against oxidative stress by regulating intracellular proline levels. Appl. Environ. Microbiol. 72: 4001-4006.

Dowdy SF, Lai KM, Weissman BE, Matsui Y, et al. (1991). The isolation and characterization of a novel cDNA demonstrating an altered mRNA level in nontumorigenic Wilms' microcell hybrid cells. Nucleic Acids Res. 19: 5763-5769.

Dresios J, Panopoulos P and Synetos D (2006). Eukaryotic ribosomal proteins lacking a eubacterial counterpart: important players in ribosomal function. Mol. Microbiol. 59: 1651-1663.

Farmer AA, Loftus TM, Mills AA, Sato KY, et al. (1994). Extreme evolutionary conservation of QM, a novel c-Jun associated transcription factor. Hum. Mol. Genet. 3: 723-728.

Green H, Canfield AE, Hillarby MC, Grant ME, et al. (2000). The ribosomal protein QM is expressed differentially during vertebrate endochondral bone development. J. Bone Miner. Res. 15: 1066-1075.

Griaznova O and Traut RR (2000). Deletion of C-terminal residues of Escherichia coli ribosomal protein L10 causes the loss of binding of one L7/L12 dimer: ribosomes with one L7/L12 dimer are active. Biochemistry 39: 4075-4081.

Imafuku I, Masaki T, Waragai M, Takeuchi S, et al. (1999). Presenilin 1 suppresses the function of c-Jun homodimers via interaction with QM/Jif-1. J. Cell Biol. 147: 121-134.

Inada H, Mukai J, Matsushima S and Tanaka T (1997). QM is a novel zinc-binding transcription regulatory protein: its binding to c-Jun is regulated by zinc ions and phosphorylation by protein kinase C. Biochem. Biophys. Res. Commun. 230: 331-334.

Kiefer F, Arnold K, Kunzli M, Bordoli L, et al. (2009). The SWISS-MODEL Repository and associated resources. Nucleic Acids Res. 37: D387-D392.

Koller HT, Klade T, Ellinger A and Breitenbach M (1996). The yeast growth control gene GRC5 is highly homologous to 
the mammalian putative tumor suppressor gene QM. Yeast 12: 53-65.

Kopp J and Schwede T (2004). The SWISS-MODEL Repository of annotated three-dimensional protein structure homology models. Nucleic Acids Res. 32: D230-D234.

Kopp J and Schwede T (2006). The SWISS-MODEL Repository: new features and functionalities. Nucleic Acids Res. 34: D315-D318.

Korostelev A, Trakhanov S, Laurberg M and Noller HF (2006). Crystal structure of a 70S ribosome-tRNA complex reveals functional interactions and rearrangements. Cell 126: 1065-1077.

Lillico SG, Mottram JC, Murphy NB and Welburn SC (2002). Characterisation of the QM gene of Trypanosoma brucei. FEMS Microbiol. Lett. 211: 123-128.

Marty I, Brugidou C, Chartier Y and Meyer Y (1993). Growth-related gene expression in Nicotiana tabacum mesophyll protoplasts. Plant J. 4: 265-278.

Melo F and Feytmans E (1998). Assessing protein structures with a non-local atomic interaction energy. J. Mol. Biol. 277: $1141-1152$.

Monteclaro FS and Vogt PK (1993). A Jun-binding protein related to a putative tumor suppressor. Proc. Natl. Acad. Sci. U.S.A. 90: 6726-6730.

Nishimura M, Kaminishi T, Kawazoe M, Shirouzu M, et al. (2007). Purification, crystallization and preliminary X-ray diffraction study of human ribosomal protein L10 core domain. Acta Crystallogr. Sect. F. Struct. Biol. Cryst. Commun. 63: 950-952.

Nishimura M, Kaminishi T, Takemoto C, Kawazoe M, et al. (2008). Crystal structure of human ribosomal protein L10 core domain reveals eukaryote-specific motifs in addition to the conserved fold. J. Mol. Biol. 377: 421-430.

Park S and Jeong DG (2006). Ribosomal protein L10 interacts with the SH3 domain and regulates GDNF-induced neurite growth in SH-SY-5y cells. J. Cell Biochem. 99: 624-634.

Saitou N and Nei M (1987). The neighbor-joining method: a new method for reconstructing phylogenetic trees. Mol. Biol. Evol. 4: 406-425.

Sambrook J and Russell DW (2001). Molecular Cloning 3. Cold Spring Harbor Laboratory Press, Cold Spring Harbor, 644-656.

Scotto-Lavino E, Du G and Frohman MA (2006). 5' end cDNA amplification using classic RACE. Nat. Protoc. 1: 25552562.

Tamura K, Dudley J, Nei M and Kumar S (2007). MEGA4: Molecular Evolutionary Genetics Analysis (MEGA) software version 4.0. Mol. Biol. Evol. 24: 1596-1599.

Tron T, Yang M, Dick FA, Schmitt ME, et al. (1995). QSR1, an essential yeast gene with a genetic relationship to a subunit of the mitochondrial cytochrome bc1 complex, is homologous to a gene implicated in eukaryotic cell differentiation. J. Biol. Chem. 270: 9961-9970.

Wen Y, Shao JZ, Pan XX and Xiang LX (2005). Molecular cloning, characterization and expression analysis of QM gene from grass carp (Ctenopharyngodon idellus) homologous to Wilms' tumor suppressor. Comp. Biochem. Physiol. B Biochem. Mol. Biol. 141: 356-365. 